\title{
Phase-Stable Millimeter-Wave Generation using Switchable Dual-Wavelength Fiber Laser
}

\author{
Hani J. Kbashi ${ }^{1 *}$, Vishal Sharma ${ }^{1}$, and Sergey Sergeyev ${ }^{1}$ \\ School of Engineering and Applied Science, Aston University, Aston Triangle, Birmingham, B4 7ET, UK. \\ "Correspondent author: h.kbashi@aston.ac.uk
}

\begin{abstract}
Dual-wavelength fiber lasers have become an attractive candidate for the last few years in the area of optical imaging, optical communication, optical sensing, microwave, and terahertz signal generation. It offers small size, inexpensive, and simple fabrication along with high scalability to the existing state-of-theart microwave-photonics networks. In this paper, we demonstrate experimentally a switchable dualwavelength fiber laser for the generation of the radio frequency signals in the millimeter band (up to 110 $\mathrm{GHz}$ ). The fiber laser is based on a nonlinear polarization rotation ring-cavity consisting of erbium-doped fiber and a high birefringence fiber of $1 \mathrm{~m}$ and $10 \mathrm{~m}$ length, respectively. By proper adjustment of the laser cavity birefringence via controlling the polarization controllers in the laser cavity, the laser output spectrum can be tuned to attain a dual-wavelength spacing in the range of $0.1 \mathrm{~nm}-0.89 \mathrm{~nm}$ to generate flexible and stable millimeter waves with an adjustable span of $12.3 \mathrm{GHz}$ to $110 \mathrm{GHz}$. The obtained results reveal the potential of the proposed laser to be used to realize different microwave-photonic systems/networks, for instance, 5G networks, internet of things, surveillance and monitoring, remote sensing, self-driving vehicles, photonics-based radar systems, meteorology and so on.
\end{abstract}

Index Terms - Dual-wavelength Fiber lasers, microwave photonics, tunable fiber lasers, 5G.

\section{Introduction.}

Switchable and tunable multi and dual-wavelength fiber lasers (DWFL) have attracted significant research interests recently due to their wide industrial applications, especially in the futuristic telecommunication industry, meteorology, automotive industry, remote sensing, LiDAR industry, and material processing related units. Such lasers have great potential and possibilities to be utilized in the implementation of futuristic $5 \mathrm{G}$ networks like a radio over fiber systems [1, 2], free space optics [3, 4], hybrid microwaveoptics systems [5], intelligent transport systems [6,7], satellite communication systems [8], LiDAR [9-11], and Terahertz $(\mathrm{THz})$ radiation applications $[12,13]$. Moreover, DWFLs are playing a significant role in generating stable millimeter $(\mathrm{mm})$ waves as compared to the conventional microwave signal generation techniques. Though the generation of $\mathrm{mm}$-wave signals has been traditionally limited exclusively within the radio frequency domain, the recent developments in photonics have augmented the opportunities of the generation of mm-waves optically. In addition, the significant benefit of this technique is the generation of phase-coherence and low phase-noise mm-wave signals with a wavelength spacing corresponding to the desired mm-wave band.

Various techniques have been explored for the generation of photonics-based microwave signals such as dual lasers with different wavelengths locked by optical phase-locking and/or injection phase-locking technique [14-15], external optical modulation [16], synchronized dual-mode laser [17], optical heterodyning techniques [15]. These techniques are not only generating non-tunable mm-waves with low phase-stability due to low phase coherence of the dual-lasers but also complex- and expensive- approaches. Nevertheless, these systems can generate good quality beat signals and have established many applications, however, they offer poor spectral purity and imperfect phase coherence. On the other hand, the fiber laser as an optical mm-wave generation source offers low phase-noise, less power consumption, narrow linewidth, and has been used as an alternative technology to overcome the weakness of the aforementioned techniques. To generate dual-wavelengths using fiber lasers, the most widely used techniques are incorporating of an optical filter into the laser's cavity, for instance, Mach Zehnder interferometers [18], fiber-based Sagnac fiber loop mirror [19] and Fabry-Pérot interferometers [20], fiber Bragg gratings (FBG) $[21,22]$ superimposed chirped FBG [23, 24], large-angle tilted FBG [25], and multimode interference (MMI) [26] or by using graphene saturable absorber [27]. But, the homogeneous gain-broadening in the gain-media causes a strong mode-competition and inherent instability. This is a big challenge to achieve a stable dual-wavelength generation using erbium-doped fiber laser (EDFL) in the $1550 \mathrm{~nm}$ wavelength region at room temperature. Moreover, it is difficult to achieve the stable dual-wavelength pulse-operation by increasing the wavelength spacing. Many techniques for mitigating the mode-competition have been 
devoted to achieve stable dual-wavelengths, for instance, using inhomogeneous loss mechanisms [20], the polarization hole burning-effect [28], cooled Er-doped fiber in liquid nitrogen (77K) [29]. Another approach based on the nonlinear properties including four-wave mixing (FWM) [30-32], stimulated Brillouin scattering $[33,34]$ and dual-wavelength phase-shifting interferometry $[35,36]$ have also been utilized to enhance the stability of the multi-wavelength fiber lasers.

However, these techniques are costly, complex, and require high-quality tunable microwave reference source. Therefore, it becomes imperative to explore some alternative simple and cost-effective switchable DWFLs having the potential to generate high phase-stable $\mathrm{mm}$-wave signals over a wide spectral range. Subsequently, in this paper, the authors demonstrate a switchable DWFL laser for generating the radio frequency signals in the mm-wave band with high quality and stability in a simple, compact and economical fashion in the laboratory environment. The proposed dual-wavelength laser is carried out by incorporating a $10 \mathrm{~m}$ of $\mathrm{HiBi}$ fiber in a ring-structure cavity along with two polarization controllers. Further to use it as a birefringent filter, we used the HiBi fiber to mitigate the inhomogeneous broadening of the Er-doped gain fiber. It is also easily integrated into the fiber-laser cavity that allows the accurate wavelength filtering to provide uniform wavelength-spacing. Moreover, the spacing between the generated dual-wavelengths can be switched over a wide range of spectral regions (ranging from $0.1 \mathrm{~nm}$ to $0.89 \mathrm{~nm}$ ) in order to generate mm-wave signals with frequency-tuning capability over the range of $\sim 12.3 \mathrm{GHz}$ to $\sim 110 \mathrm{GHz}$ in a tunable step of $\sim 10 \mathrm{GHz}$. This can be attained by beating the two wavelengths via a simple adjustment of the state of polarization (SOP) in the laser-cavity. The presented work is structured as: Section 1 describes the earlier reported work for different techniques used to generate $\mathrm{mm}$ waves; Section 2 describes the experimental setup of the proposed DWFL laser; Section 3 discusses the outcomes of the proposed DWFL and is followed by the concluding part as Section 4 .

\section{Experiment Setup.}

A simple and compact configuration of the proposed switchable DWFL set-up in the lab environment is illustrated in Fig. 1(a). A 980-nm laser diode is used as a pump source to pump a 1-m single-mode erbiumdoped active fiber (Liekki Er80-8/125) via a wavelength-division-multiplexer (WDM-980/1550 nm). Two polarization controllers (PCs) are used inside the cavity to perform the nonlinear polarization rotation (NPR). Also, the set-up includes a polarization-independent optical isolator to ensure unidirectional propagation. The laser-cavity also contains a bow-tie geometry of high birefringence (HiBi) fiber of 10-m length with a numerical aperture of 0.125. The core and cladding diameters of HiBi fiber are $8.5 \mu \mathrm{m}$ and $125 \mu \mathrm{m}$, respectively. A 90:10 fused fiber output coupler is used to redirect the $90 \%$ of the signal power to the laser cavity and $10 \%$ of it for the spectral and temporal analysis. Therefore, the overall cavity length is measured as $20.1 \mathrm{~m}$.

The authors used HiBi fiber as an optical filter inside the laser cavity to enhance the dual-wavelength stability by preserving the state of polarization (SOP). This fiber boasts an extreme birefringence with the polarization beat-lengths approaching $2.5 \mathrm{~mm}$. Hence, the laser net cavity-birefringence is the entirety of HiBi and SMF fibers birefringence that have been controlled using the two PCs in order to attain a stable lasing action in/out of the lab environment. Also, HiBi fiber can be easily incorporated into the laser-cavity and offers an accurate wavelengths-filtering over a broad spectral-band (provides a usable bandwidth of 100 $\mathrm{nm}$ ) to attain a uniform channel-spacing in comparison with the existing filtering techniques. However, the existing filtering techniques in the literature experience some limitations including complex fabrication, non-tunable, and highly sensitive to the environment.

Fig. 1(b) shows the measured output power from $10 \%$ of the output coupler vs pump power. Further, the laser output is observed by using a photo-detector (InGaAsUDP-15-IR-2_FC) with a bandwidth of $17 \mathrm{GHz}$ connected to a $2.5 \mathrm{GHz}$ sampling oscilloscope (Tektronix DPO7254). The polarization properties have been studied using a commercial polarimeter (Thorlabs IPM5300) with $1 \mu$ s resolution with an interval of $1 \mathrm{~ms}$ (25-40000 round-trips) to measure the normalized Stokes parameters $S_{1}, S_{2}, S_{3}$, and degree of polarization $(D O P)$. The Intra-cavity PCs are tuned to study the polarization dynamics. An optical spectrum analyzer (Yokogawa AQ6317B) with a maximum resolution of $20 \mathrm{pm}$, and radio frequency spectrum analyzer ( $F S V$ Rohde Schwarz) were used to observe the optical and the RF spectrums respectively. 
a)

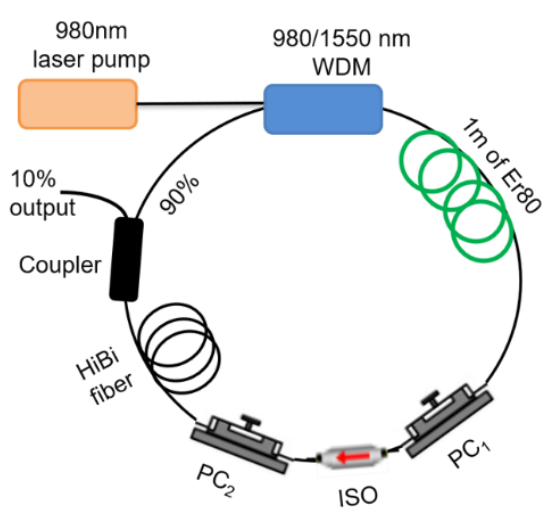

b)

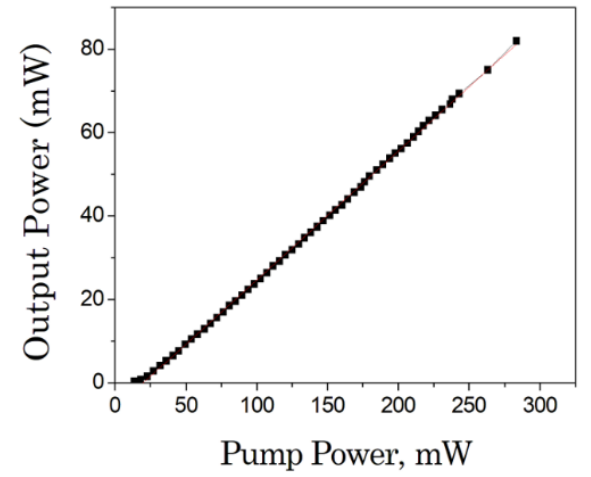

Fig. 1. (a) The experimental setup of the proposed switchable dual-wavelength fiber laser; (b) the measured output power from the $10 \%$ of the output coupler vs pump power.

\section{Results and Discussions.}

Dual-wavelength output spectra of the demonstrated experiment are shown in Fig. 2 at a pump power of 20 $\mathrm{mW}$. By the adjustment of the laser net-birefringent cavity via a controlled tuning of the PCs, a dualwavelength lasing is attained with a wavelength spacing of $0.1 \mathrm{~nm}$ as shown in Fig. 2(a) that analogous to $12.3 \mathrm{GHz}$. It is worth noting that the dual-wavelength output is located at $1560.0 \mathrm{~nm}$ and $1560.1 \mathrm{~nm}$, with a very narrow linewidth of $0.125 \mathrm{~nm}$ and $0.13 \mathrm{~nm}$, respectively. By tuning the two PCs carefully, the twowavelength lasing peaks are shifted and the wavelength-spacing is increased from $0.1 \mathrm{~nm}$ to $0.89 \mathrm{~nm}$ sequentially which corresponds to the beating frequency in the span of $12.3 \mathrm{GHz}$ to a $110 \mathrm{GHz}$ as shown in Fig. 2(b-g) over a span of $4 \mathrm{~nm}$. The largest wavelength spacing is $0.89 \mathrm{~nm}$ which corresponds to a beating frequency of $110 \mathrm{GHz}$, which is achieved with the demonstrated experiment. And, the two laser peaks are located at $1559.54 \mathrm{~nm}$ and $1560.43 \mathrm{~nm}$ with a narrow linewidth of $0.12 \mathrm{~nm}$ and $0.1 \mathrm{~nm}$, respectively. Moreover, the adjustment of the PCs tunes the HiBi based mode-beating filter to control the total cavity birefringence. This leads to generating a dual-wavelength lasing action at the low pump-power with tunable wavelength-spacing and hence, increases the free spectral-range of the HiBi filter. It is apparent from Fig. 2 that the generated dual-wavelength has a small and slow amplitude variation which may be attributed to the wavelength dependence of the phase-shifts between the two PCs. Further, the beat signals corresponding to the wavelength-spacing of the demonstrated laser output are measured after the photo-detection using the computer-based Optisystem ${ }^{\mathrm{TM}}$ photonic software as shown in Fig. 3. The RF spectra show the beat signals of the last four highest frequencies $56 \mathrm{GHz}, 77 \mathrm{GHz}, 86 \mathrm{GHz}$, and $110 \mathrm{GHz}$ corresponding to the wavelength-spacing $0.48 \mathrm{~nm}, 0.63 \mathrm{~nm}, 0.7 \mathrm{~nm}$, and $0.89 \mathrm{~nm}$ respectively. The outcomes reveal that by a controlled tuning of the laser cavity birefringence, the demonstrated DWFL laser has the ability to generate such high carrier frequencies that can be used in different $5 \mathrm{G}$ networks and LiDAR-based applications.

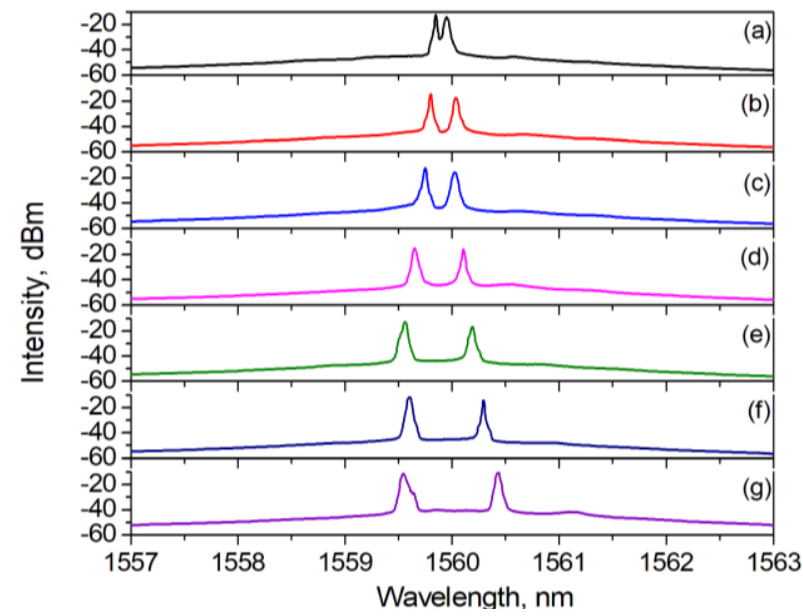

Fig. 2. Switchable DWFL based generation of waveforms with wavelength-spacing of (a) 0.1 nm; (b) $0.2 \mathrm{~nm}$; (c) $0.29 \mathrm{~nm}$; (d) $0.48 \mathrm{~nm}$; (e) 0.63 $\mathrm{nm}$; (f) $0.7 \mathrm{~nm}$; and (g) $0.89 \mathrm{~nm}$.
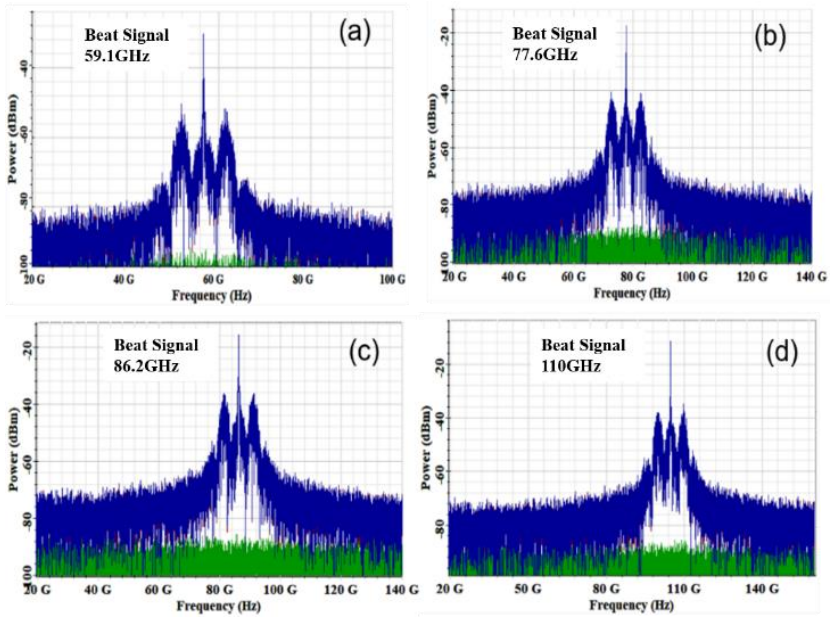

Fig. 3. Measurements of Beat signals after photo-detection at (a) $59.1 \mathrm{GHz}$, (b) $77.6 \mathrm{GHz}$, (c) $86.2 \mathrm{GHz}$, and (d) 110 GHz. 
It is also observed from the carried out experiment that the center-wavelength of the laser-cavity is fixed around $1560 \mathrm{~nm}$, while the passband spacing between the two defined wavelengths is varied from $\approx 0.1 \mathrm{~nm}$ to $\approx 0.89 \mathrm{~nm}$ via a controlled adjustment of the two PCs. Moreover, the center-wavelength is observed well consistent with the passband spacing defined by the HiBi fiber. Upon entering through the HiBi fiber, it preserves the state-of-polarization of these emitted wavelengths at the linearly polarized state inside the laser-cavity. The fine-tuning of the PCs produce the output of the demonstrated dual-wavelength laser with nearly identical peaks with a maximum power of $\approx-11.48 \mathrm{dBm}$ and $\approx-10.64 \mathrm{dBm}$ as shown in Fig. 2 . It is also observed that the distribution of the dual-wavelength in the laser-cavity is highly dependent on the polarization states of the two emitted wavelengths.

Further, to investigate the stability of the generated dual-wavelength lasing, the authors have measured the output spectra over 60 min as illustrated in Fig. 4(a), with an interval scanning of 10 minutes. It is apparent from Fig. 4(a) that the optical-spectra shows a high stable laser-output without displaying any wavelength-shift during the observation period of measurements. Also, a high optical signal-to-noise ratio $(\approx 40 \mathrm{~dB})$ for both the peaks is measured. The dual-wavelength modes are observed at $1559.5 \mathrm{~nm}$ and 1560.35 respectively with a wavelength spacing of $0.85 \mathrm{~nm}$. The wavelength fluctuations of $0.04 \mathrm{~nm}$ and $0.03 \mathrm{~nm}$ along with their power fluctuations of $0.46 \mathrm{~dB}$ and $0.32 \mathrm{~dB}$, respectively, are measured over the observation period of 60 minutes which claims a good power uniformity and stability. The stable dualwavelength operation may be attributed to the upholding of SOP using HiBi fiber and to effectively suppress the mode-competition of the homogenous line-broadening and cross-gain saturation in the Er-doped fiber. However, the insertion of the PCs with HiBi into the cavity provides a wavelength-dependent polarization rotation and varies the SOPs across the multiple wavelengths. The authors also believe that both the PCs and HiBi together help to achieve an optimum amplification performance at low pump-power by managing the SOPs to attain a linearly polarized output. This further leads to a stable and switchable DWFL laser in the laboratory environment. Further, the beat length $\left(\mathrm{L}_{b}\right)$ is calculated (Fig. 4(b)) as a function of the dualwavelength spacing that fluctuates from $\approx 1.3 \mathrm{~mm}$ to $\approx 12 \mathrm{~mm}$. This computes the difference between the two orthogonal polarization refractive-indices and is measured in the range of minima to maxima as $1.35 \times 10^{-4}$ to $1.2 \times 10^{-3}$ in this experiment.
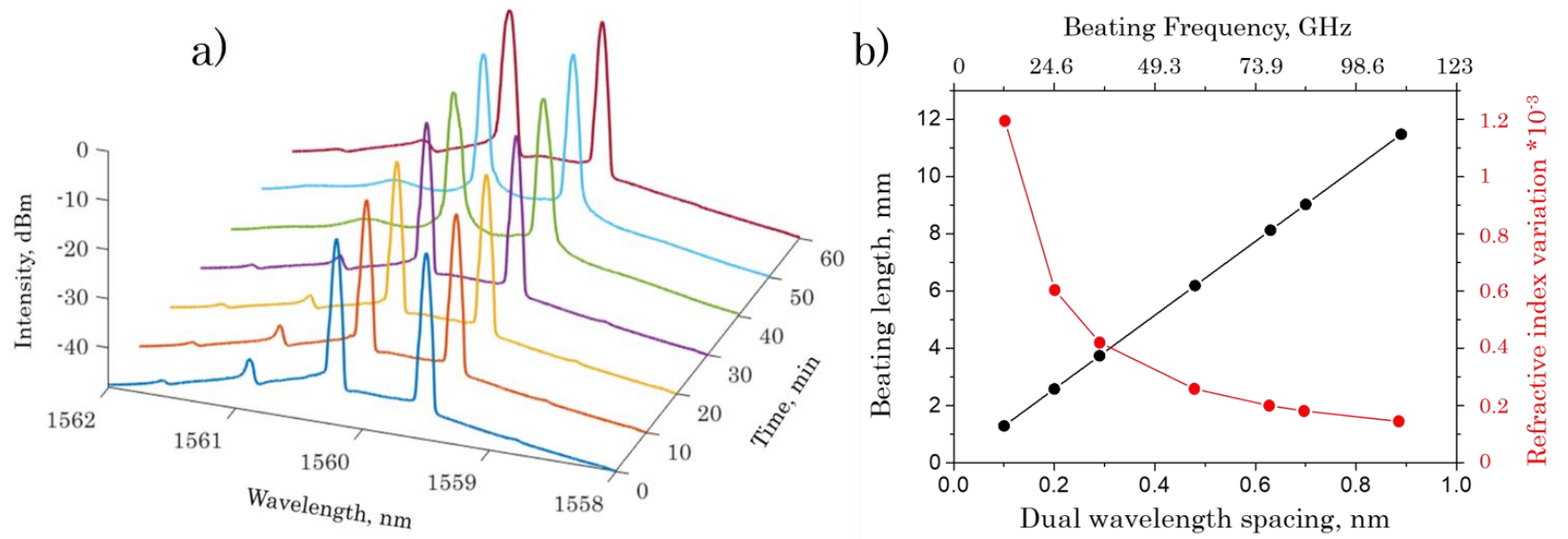

Fig. 4. (a) The stability spectra of the switchable DWFL-based generation with wavelength-spacing of $0.85 \mathrm{~nm}$ at observation time of 60 minutes; (b) the beat-length and the two orthogonal polarization refractive-indices variation as a function of different dual-wavelength spacing.

Therefore, the authors have characterized the SOP dynamics using a polarimeter by measuring the normalized Stokes parameters, i.e. $S_{1}, S_{2}, S_{3}$, and the degree of polarization (DOP). Fig. 5 illustrates a stable polarization operation for most of the observed dual-wavelength regimes (as for $0.1 \mathrm{~nm}-0.48 \mathrm{~nm}$ ) in the Poincare sphere with the axis defined by the three Stokes parameters. These stable and slow polarization dynamics indicate a high coherent coupling between the two orthogonal polarization which leads to producing a single-pulse (mode-locked) regime due to the synchronization of two orthogonal SOPs. Furthermore, in the cases of $0.7 \mathrm{~nm}$ and $0.89 \mathrm{~nm}$, it is observed that the laser system starts desynchronizing the two orthogonal SOPs at $0.7 \mathrm{~nm}$ while it attains a complex chaotic regime at $0.89 \mathrm{~nm}$ as shown in Fig. 5 (a). This indicates an incoherent coupling between the two orthogonal polarizations as the DOP is decreased to less than $40 \%$ (Fig. 5(b)). Moreover, this synchronization and coupling behavior can be described via the vector resonance multimode instability [37]. 

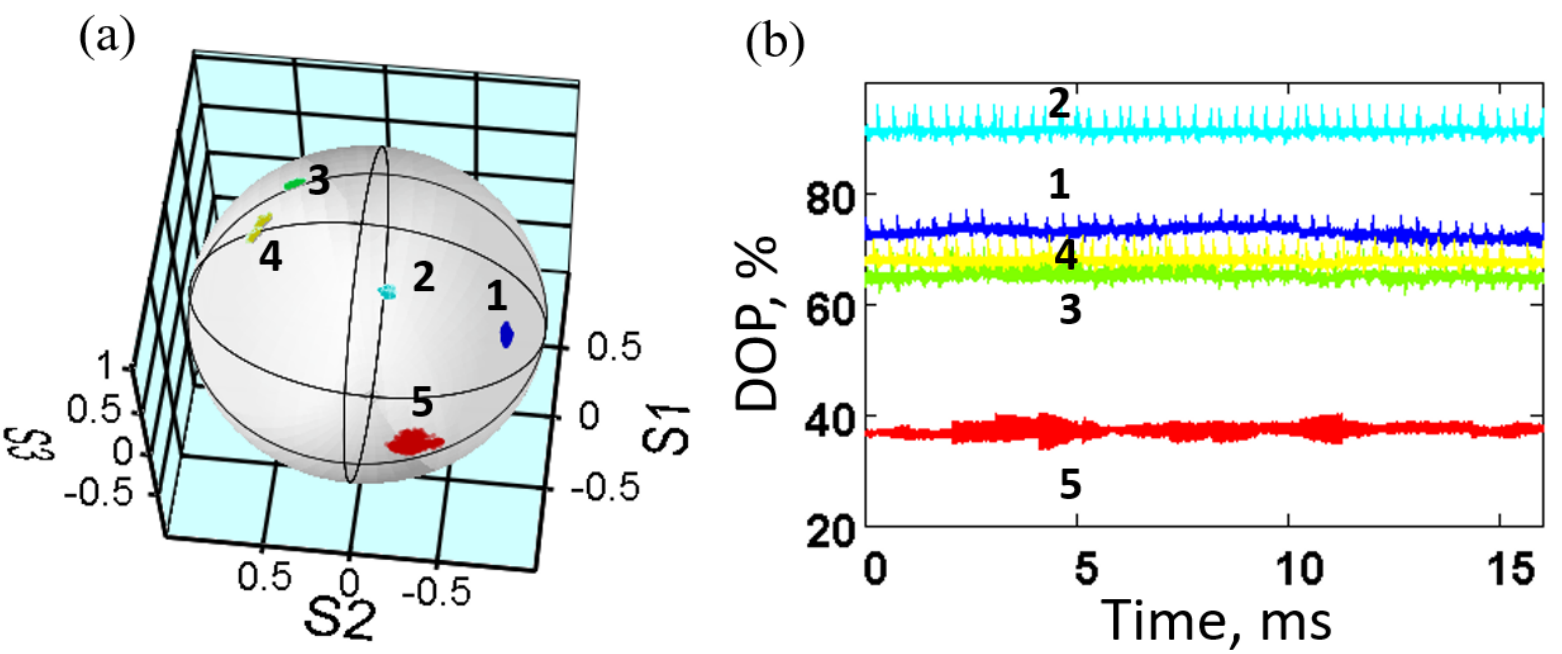

Fig. 5. (a) the SOP; (b) DOP of the switchable dual-wavelength fiber laser-based generation at different wavelength spacing, $1-0.1 \mathrm{~nm} ; 2-0.29 \mathrm{~nm} ; 3-0.48 \mathrm{~nm} ; 4-0.7 \mathrm{~nm}$; and $5-0.89 \mathrm{~nm}$.

Furthermore, the authors have measured the corresponding radio frequency (RF) spectrum, with a resolution bandwidth of $3 \mathrm{~Hz}$ as shown in Fig. 6(a). When the pump power increases to $20 \mathrm{~mW}$, it is observed that the self-started pulses occur with a repetition rate of $9.945 \mathrm{MHz}$. As shown in Fig. 6(a), the central frequency is $9.945 \mathrm{MHz}$ with a spectral width of $3.46 \mathrm{kHz}$, which agrees with the pulse repetition rate interval of 100.5 ns shown in Fig. 6 (b). The signal-to-noise-ratio $(S N R)$ is measured as $\approx 70 \mathrm{~dB}$, indicating the high stability of the laser output. Fig. 6(c) shows the measured pulse duration of about $1 \mathrm{ps}$ at the stability of the switchable DWFL-based generation at $0.85 \mathrm{~nm}$.

(a)

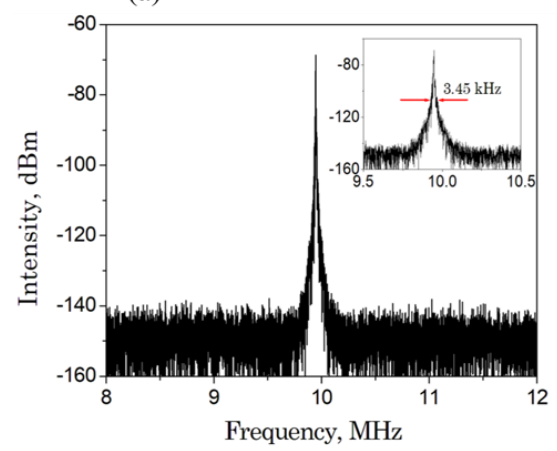

(b)

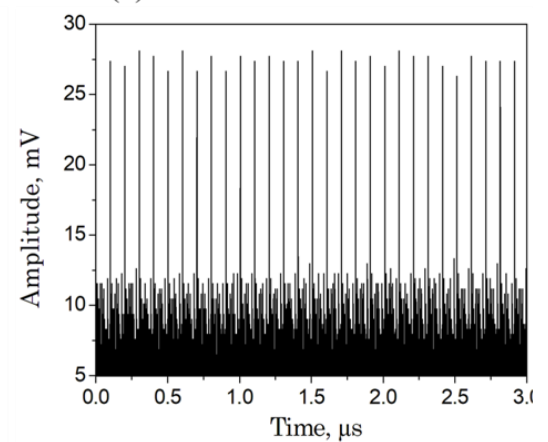

c)

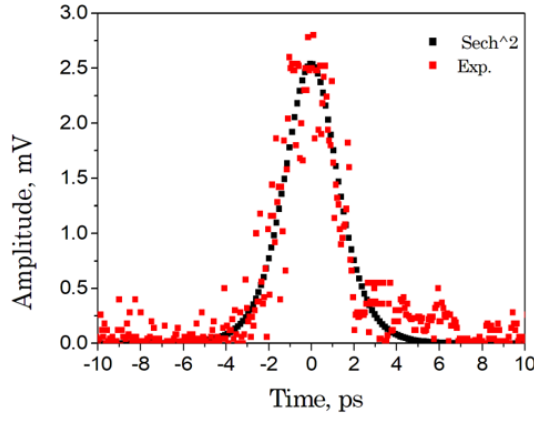

Fig. 6(a). The frequency spacing between the neighboring beat-signals is $9.945 \mathrm{MHz}$, (b) corresponding time interval of $100.5 \mathrm{~ns}$ which corresponds to a cavity length of about $20.1 \mathrm{~m}$; (c) the measured pulse duration at the stability of the switchable DWFL-based generation at $0.85 \mathrm{~nm}$.

\section{Conclusions.}

We demonstrated experimentally a simple, economical, highly phase-stable, easily scalable, and a switchable dual-wavelength fiber laser successfully for the generation of millimeter waves over a wide frequency range in mm-wave band. A controlled and fine-tuning of the laser cavity birefringence via a piece of HiBi fiber in conjunction with two PCs, the generated dual-wavelength can be switched over a broad wavelength-spacing range, i.e. $0.1 \mathrm{~nm}$ to $0.89 \mathrm{~nm}$ which corresponds to the beating frequency of $\approx 12.3 \mathrm{GHz}$ to $\approx 110 \mathrm{GHz}$. The authors believe that the proposed dual-wavelength fiber laser could have potential opportunities in the development and implementation of the futuristic 5G and LiDAR transmission systems along with some state-of-the-art meteorology and high-precision spectroscopy applications.

\section{ACKNOWLEDGMENT.}

This work is carried out in Aston Institute of Photonic Technologies, School of Engineering and Applied Sciences, Aston University, Birmingham, UK and is supported by European Union sponsored H2020-MSCA-IF-EF-ST project no: 840267. 


\section{References.}

[1] Moussa El Yahyaoui, Ali El Moussati, Ghaïs El Zein, KamelHaddadi, "New millimeter wave generation scheme for MIMO-OFDM based Radio-over-Fiber system," Opt Comm 2019, 442, 101-105.

[2] Cheng-TingTsai, Chien-Cheng Li, Chi-Hsiang Lin , Chun-Ting Lin , SienChi and Gong-Ru Lin. Long-reach 60-GHz MMWoF link with free-running laser diodes beating. Sci Rep 2018, 8, 13711.

[3] Z. Jia, J. Yu, G. Ellinas, and G. K. Chang. Key enabling technologies for optical-wireless networks: optical millimeter-wave generation, wavelength resuse and architecture. J. Lightwave Technol 2007, 25, 3452-3471.

[4] J. Yu, G. K. Chang, Z. Jia, A. Chowdhury, M. F. Huang, H. C. Chien, Y. T. Hsueh, W. Jian, C. Lieu, and Z. Dong. Cost-effective optical millimeter technologies and field demonstrations for very high throughput wireless-over-fibre access systems. J. Lightwave Technol 2010 , 28, 2376-2397.

[5] Dao-Quan Zhu and Peng-Bo Li. Preparation of entangled states of microwave photons in a hybrid system via the electro-optic effect. Opt Express 2017, 25.

[6] Anum Khattak, Gerard Tatel and LiWei. Tuneable and Switchable Erbium-Doped Fibre Laser Using a Multimode-Fibre Based Filter. Appl. Sci 2018, 8, 1135 .

[7] P. Papadimitratos, A. D. La Fortelle, K. Evenssen, R. Brignolo and S. Cosenza. Vehicular communication systems: Enabling technologies, applications, and future outlook on intelligent transportation. in IEEE Communications Magazine 2009, 47, 84-95.

[8] M. Jia, X. Gu, Q. Guo, W. Xiang and N. Zhang. Broadband Hybrid Satellite -Terrestrial Communication Systems Based on Cognitive Radio toward 5G. IEEE Wirel Commun 2016, 23, 96 -106.

[9] D. Bouteyre, G. Canat, M. Valla, B. Augère, C. Besson, D. Goular, L. Lombard, J.-P. Cariou, A. Durecu, D. Fleury, L. Bricteux, S Brousmiche, S. Lugan and B. Macq. Pulsed $1.5 \mu \mathrm{m}$ lidar for axial aircraft wake vortex detection based on high brightness large-core fibre amplifier. IEEE J. Sel. Top. Quantum Electron 2009, 15, 441-450.

[10] S. Gao, M. O’Sullivan, and R. Hui. Complex-optical-field Lidar system for range and vector velocity measurement. Opt Express 2012, 20, 25867-25875.

[11] P. Adany, C. Allen and R. Hui. Chirped lidar using simplified homodyne detection. J of Lightwave technol 2009, 27, $3351-3357$.

[12] Yue Yang, Jie Li, Jining Li, Jin Huang, Yating Zhang, Lanju Liang, Jianquan Yao. Plasmon-induced reflection metasurface with dual-mode modulation for multi-functional THz devices. Opt Lasers in Eng 2020, 127, 105969.

[13] Yuying Lu, Maosheng Yang, Zhang Zhang, Lanju Liang, Jining Li, and Jianquan Yao. Dual-wavelength terahertz sensing based on anisotropic Fano resonance metamaterials. Appl Opt 2019, 58, 1667-1674.

[14] L. Goldberg, H. F. Taylor, J. F. Weller, and D. M. Bloom. Microwave signal generation with injection locked laser-diodes. Electron Lett 1983, 19, 491-493.

[15] R. T. Ramos and A. J. Seeds. Fast heterodyne optical phase-lock loop using double quantum-well laser diodes. Electron Lett 1992, 28, 8283.

[16] G. H. Qi, J. P. Yao, J. Seregelyi, S. Paquet, and C. Bélisle. Generation and distribution of a wide-band continuously tuneable millimeterwave signal with an optical external modulation technique. IEEE Trans Microwave Theory Tech 2005, 53, 3090-3097.

[17] Wen Qi Zhang David G. Lancaster, Tanya M. Monro \& Shahraam Afshar Vahid. Synchronised dual-wavelength mode-locking in waveguide lasers. Sci Rep 2018, 8, 7821.

[18] N. Yan, X. Han, P. Chang, L. Huang, F. Gao, X. Yu, W, Zhang, Ze Zhang, G. Zhang, J. Xu. Tuneable dual-wavelength fibre laser with unique gain system based on in-fibre acousto-optic Mach-Zehnder interferometer. Opt Express 2017, 2527609.

[19] MA. Mirza, G. Stewart. Theory and design of a simple tunable Sagnac loop filter for multi-wavelength fibre lasers. Appl Opt 2008, 47:52425252.

[20] S. Pan, C. Lou, and Y. Gao. Multiwavelength erbium-doped fiber laser based on inhomogeneous loss mechanism by use of a highly nonlinear fiber and a Fabry-Perot filter. Opt. Express 2006 14, 1113-1118.

[21] S. Longhi, M. Marano, P. Laporta, O. Svelto. Single- and dual-wavelength pulse train generation in frequency-modulated Er-Yb lasers up to $20 \mathrm{GHz}$. Opt Lasers in Eng 2003, 39, 269-275.

[22] Xueming Liu, Xiufeng Yang, Fuyun Lu, Junhong Ng, Xiaoqun Zhou, and Chao Lu. Stable and uniform dual-wavelength erbium- doped fibre laser based on fibre Bragg gratings and photonic crystal fibre. Opt Express 2005, 13, 142-147.

[23] M. Li, X. Chen, T. Fujii, Y. Kudo, H. Li, and Y. Painchaud. Multiwavelength fiber laser based on the utilization of a phase-shifted phaseonly sampled fiber Bragg grating. Opt Lett 2009, 34, 1717.

[24] J. Yang, S. C. Tjin, and N. Q. Ngo. Multiwavelength Tunable Fiber Ring Laser Based on Sampled Chirp Fiber Bragg Grating. . IEEE Photon Technol Lett 2004, 16, 1026-1028.

[25] C. Mou, P. Saffari, H. Fu, K. Zhou, L. Zhang, and I. Bennion. Single- and dual-wavelength switchable erbium-doped fiber ring laser based on intracavity polarization selective tilted fiber gratings. Appl Opt 2009, 48, 3455.

[26] A. Khattak, G. Tatel, and L. Wei. Tunable and Switchable Erbium-Doped Fiber Laser Using a Multimode-Fiber Based Filter. Appl. Sci $2018,8,1135$.

[27] Jingjuan Zhou, Aiping Luo2 Zhichao Luo, Xudong Wang, Xinhuan Feng, and Bai-ou Guan. Dual-wavelength single-longitudinal-mode fibre laser with switchable wavelength spacing based on a graphene saturable absorber. Photon Res 2015, 3 A21-A24.

[28] Y. Lian, G. Ren, B. Zhu, Y. Gao, W. Jian, W. Ren, and S. Jian. Switchable multiwavelength fiber laser using erbium-doped twin-core iber and nonlinear polarization rotation. Laser Phys. Lett. 2017, 14, 055101.

[29] S. Yamashita and K. Hotate. Multiwavelength erbium-doped fiber laser using intracavity etalon and cooled by liquid nitrogen. Electron. Lett $1996,32,1298-1299$.

[30] Wang, Q., Rideout, H., Zeng, F. \& Yao, J. Millimeter-wave frequency tripling based on four-wave mixing in a semiconductor optical amplifier. IEEE Photon Technol Lett 2006, 18, 2460-2462.

[31] Shih, P.-T. et al. Optical millimeter-wave signal generation via frequency 12-tupling. J Lightwave Technol $2010,28,71-78$.

[32] Li, M., Chen, H., Yin, F., Chen, M. \& Xie, S. Full-duplex 60-GHz RoF system with optical local oscillating carrier distribution scheme based on FWM effect in SOA. IEEE Photon Technol Lett 2009, 21, 1716-1718.

[33] Schneider, T., Junker, M. \& Lauterbach, K.-U. Theoretical and experimental investigation of Brillouin scattering for the generation of millimeter waves. JOSA B 2006, 23, 1012-1019.

[34] Li, J., Lee, H. \& Vahala, K. J. Microwave synthesizer using an on-chip Brillouin oscillator. Nat comm $2013,4,3097$.

[35] Qian Liu, Lulu Li, Hui Zhang, Wen Huang, Xiaobin Yue. Simultaneous dual-wavelength phase-shifting interferometry for surface topography measurement. Opt Lasers in Eng 2020, 124, 105813.

[36] Tong Guo, Feng Li, Jinping Chen, Xing Fu, Xiaotang Hu. Mult-wavelength phase-shifting interferometry for micro-structures measurement based on color image processing in white light interference. Opt Lasers in Eng 2016, 82, 41-47.

[37] S. V. Sergeyev, H. Kbashi, N. Tarasov, Yu. Loiko, and S. A. Kolpakov. Vector-Resonance-Multimode Instability. Phys Rev Lett 2017; 118, 033904. 